\title{
The TAx4 experiment
}

\author{
Eiji Kido* for the Telescope Array Collaboration \\ Institute for Cosmic Ray Research, University of Tokyo, 5-1-5 Kashiwanoha, Chiba 277-8582, \\ Japan \\ E-mail: ekidodicrr.u-tokyo.ac.jp
}

The Telescope Array (TA) experiment consists of a surface detector (SD) array covering $700 \mathrm{~km}^{2}$ in area and three fluorescence detector (FD) stations and explores the origin of ultra-high-energy cosmic rays. We found the evidence of a hotspot in the arrival directions of cosmic rays with energies above $57 \mathrm{EeV}$. New SDs and FDs are planned to be constructed for the TAx4 experiment to cover 4 times larger area than TA to observe cosmic rays especially with the highest energies using high statistics. This project is expected to clarify not only the source of the hotspot but also the energy spectrum and the composition at the highest energies. The five-year proposal for TAx4 SD was accepted in the spring of 2015. The proposal for constructing 2 FD stations was also accepted in 2016. The current status and the future prospects of the TAx4 experiment are shown.

35th International Cosmic Ray Conference - ICRC2017

10-20 July, 2017

Bexco, Busan, Korea

${ }^{*}$ Speaker. 


\section{Introduction}

Telescope Array (TA) is the largest cosmic-ray observatory in the Northern Hemisphere. The TA experiment has 507 surface detectors (SDs) on a square grid with $1.2 \mathrm{~km}$ spacing covering approximately $700 \mathrm{~km}^{2}$. The SDs are surrounded by three fluorescence detector (FD) stations (12, 12 and 14 telescopes). The duty cycle of the SD array is greater than $95 \%$ throughout 5-year observation period, whereas the FD duty cycle is about $10 \%$ because the data is taken only on moonless clear nights. The left figure of Fig.W is the map with locations of the detectors of TA. The latitudes and the longitudes of the locations of the detectors are around $39.30^{\circ} \mathrm{N}$ and $112.91^{\circ} \mathrm{W}$ in Utah in the USA.

The hotspot was observed in the arrival directions of 72 cosmic rays with energies above 57 $\mathrm{EeV}$ [四]. The data used in the analysis was obtained by observing with TA SD for 5 years. The chance probability to exceed the obtained maximum significance $(5.1 \sigma)$ in an isotropy sky is estimated to be $3.4 \sigma$ in the published paper.

TAx4 SD array was designed to study cosmic rays with energies especially above $57 \mathrm{EeV}$ by accelerating the pace of the data collection. The spacing of TAx4 SD array is more sparse than TA SD to obtain larger detection area. The spacing of the array is $2.08 \mathrm{~km}$. Cosmic rays with higher energies make larger number of SDs triggered and the sparse array does not lose the quality of event reconstructions very much at the higher energies. 500 TAx4 SDs cover about 3 times larger area than 507 TA SDs and the combined coverage of TAx4 SDs with TA SDs is about $3000 \mathrm{~km}^{2}$. Fig.W shows the actual planned locations of TAx4 detectors. This coverage is comparable to that of the Pierre Auger Observatory which has the largest coverage in the Southern Hemisphere. 2 FD stations will also be constructed and the field of view of FDs will cover the detection area of SDs. The SD and FD hybrid observation will provide the information of the mass composition of cosmic rays.

\section{The Design and the Performance of TAx4 SDs}

The basic design of TAx4 SD is same as TA SD ["]]. Each SD is composed of 2 layers of $1.2 \mathrm{~cm}$ thick, $3 \mathrm{~m}^{2}$ plastic scintillators and the scintillation light is read out by wave length shift (WLS) fibers and photomultiplier tubes (PMTs). Coincidence signals of 2 layers of scintillators are taken and the signals are calibrated with single muons. The taken data in each SD is collected by electronics in the communication towers using $2.4 \mathrm{GHz}$ wireless LAN communication.

The arrangement of WLS fibers (Y-11 Kurary) was changed from TA SD. The quantum efficiency of PMT (R8619 Hamamatsu) for TAx4 SDs is about twice as high as that for TA SDs and the smaller number of photons from fibers is allowed for TAx4. Eventually about $67 \%$ of WLS fibers were reduced from TA SD. Fig. 1 shows the outlook of TAx4 SD and the schematic view of the scintillator box.

The performance of TAx4 SD array was estimated using similar air shower simulations and the detector simulations to TA SDs. We expect about $25 \%$ energy resolution, $2.2 \mathrm{deg}$. angular resolution and $95 \%$ reconstruction efficiency for $\mathrm{E}>57 \mathrm{EeV}$ cosmic rays. Table $\mathrm{W}$ shows the comparison of the performance of TAx4 SD with TA SD. The values of TAx4 SD in the table do not include array boundary events. The data equivalent of 19 years TA SD is planned to be taken 
with both TAx4 and TA SD array. In designing TAx4 SD array, the significance of the hotspot was expected to be more than $5 \sigma$ with the data using the estimated performance.

Table 1: Comparison of the performance of TAx 4 SD with TA SD for cosmic rays with $\mathrm{E}>57 \mathrm{EeV}$ SD array $\quad$ Angular Resolution (deg.) $\quad$ Energy Resolution (\%) $\quad$ Reconstruction Efficiency (\%)

$\begin{array}{llll}\text { TA SD } & 1.0 & 15 & 99\end{array}$

$\begin{array}{llll}\text { TAx4 SD } & 2.2 & 25 & 95\end{array}$

\section{Calibration of TAx4 SDs}

We performed the calibration using single atmospheric muons for 173 assembled SDs. Fig.[3] shows an example of the typical charge distribution of coincidence signals of 2 layers of scintillators. Convoluted Landau and Gaussian fitting function is practically used for the evaluation of the distribution to give a good fit to the asymmetric distribution. The obtained most probable (MP) value of the Landau distribution of single muons corresponds to 19 photo electrons in average. The root mean square of the number of photo electrons is 3.3. The obtained same parameters by TA SDs were 24.6 and 7.2 respectively. The number of photo electrons from TAx 4 SDs are within the range obtained by TA SDs. Fig. . 1 shows all of obtained MP values of TAx4 SDs and the dependence of the production number of SDs.

4 LEDs are instrumented in each SD. 2 LEDs are instrumented for the calibration of linearity of the PMT for the upper layer of scintillators. 2 LEDs are for the lower layer. Linearity of the output current of all PMTs was measured with the LEDs. Fig.[1 shows the measured typical nonlinearity of the PMT of TA and TAx4 SDs. When the non-linearity of the current is about 5\%, the typical output current of PMTs for TAx4 SDs is about $50 \mathrm{~mA}$ and the typical output current of PMTs for TA SDs is about $25 \mathrm{~mA}$. The readout SD electronics saturates at $40 \mathrm{~mA}$ and this saturation is earlier than PMTs for TAx4 SDs.

The internal clock of each SD electronics is calibrated using 1PPS of the GPS timing receiver (M12M i-Lotus) which is instrumented on the SD electronics. The accuracy of the calibration was checked in the following way. One test pulse from a function generator was divided and the same divided pulses were input to some SD electronics and the difference of the trigger timings between SD electronics was measured. In this test, one particular SD electronics and a GPS receiver with the serial number RD4569 were used as the standard reference. In each test, the GPS timing receivers on SD electronics were replaced except for the reference SD electronics and the relative timings to the reference were measured. The signal of GPS antenna was also divided and the same signals were input to timing receivers. The same data acquisition program of the electronics as used for the air shower events was used in this test. The relative trigger timings of 93 different GPS timing receivers compared with the reference GPS timing receiver were measured.

The obtained difference of the trigger timings is summarized in Fig.6. The average of the relative timing offset of 93 receivers to the reference was $0.6 \mathrm{~ns}$ and the root mean square was 4.9 ns. The largest timing offset was $16.7 \mathrm{~ns}$. The fluctuation of the timing offset was about $13.3 \mathrm{~ns}$ and this fluctuation did not change much more than $0.2 \mathrm{~ns}$ even if the timing receivers were replaced. This result suggests that the resolution of each timing receiver is about $13.3 / \sqrt{2}=9.4$ ns. This accuracy is same as that of TA SDs and good enough for the event reconstructions. 


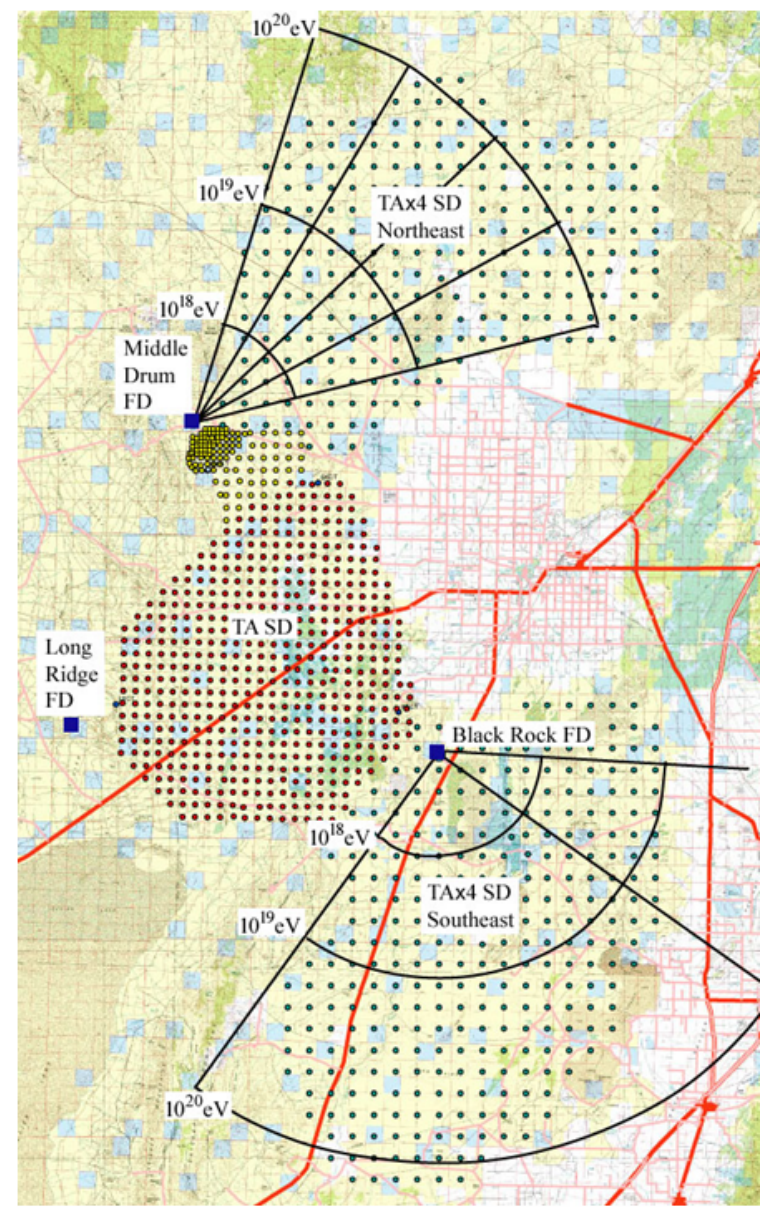

Figure 1: This map shows the overview of the TA site. Each green circle in the northeast and southeast corresponds to the planned location of each TAx 4 SD. The spacing of TAx 4 SD is $2.08 \mathrm{~km}$. The red circle in the west shows the location of TA SD. The spacing of TA SD is $1.2 \mathrm{~km}$. The 2 fan shapes drawn with black lines describe the expected field of view from TAx4 FDs. Four telescopes of FD will be built in the north Middle Drum site and 8 telescopes of FD will be built in the south Black Rock site. The overlap of the locations of SD and the field view of FD enables SD and FD hybrid observation.

\section{Summary and Future Prospects}

The assembly of first $173 \mathrm{TAx} 4 \mathrm{SD}$ is in progress. We found that the distribution of singlemuon response for TAx4 SDs is tighter than that for TA SDs. We also found that PMTs of TAx4 SDs have wider linear range than TA SDs and the ADC of the electronics limits the range. The accuracy of GPS timing receivers of TAx4 SDs was checked and it is comparable to that of TA SDs.

New 77 TAx4 SDs is planned to be assembled and the staking of the TAx4 SD site will be 

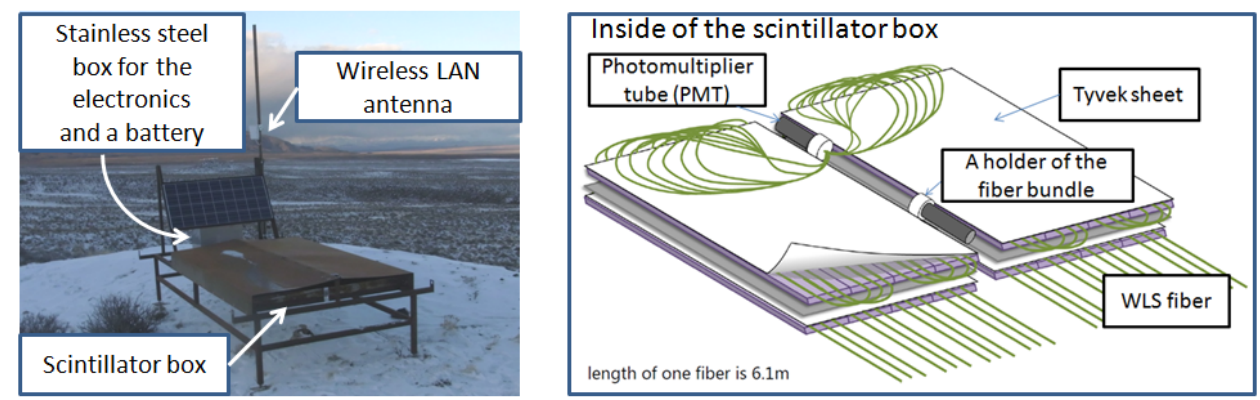

Figure 2: The left figure shows the picture of the outlook of TAx4 SD. The scintillator box is covered with the roof as in the figure. The wireless LAN antenna is fixed with the antenna pole and the direction is adjusted to the communication tower. The solar panel charges the battery in the stainless steel box and supplies power to the electronics and other devices. The right figure shows the schematic view inside of the scintillator box. Plastic scintillators and WLS fibers are covered with 2 tyvek sheets. The length of each fiber is $6.1 \mathrm{~m}$ and the length between fibers is $4 \mathrm{~cm}$ on the plastic scintillator. The each fiber bundle is composed of 56 edges of 28 fibers. The fiber bundle is polished and connected to the surface of the PMT with optical grease.

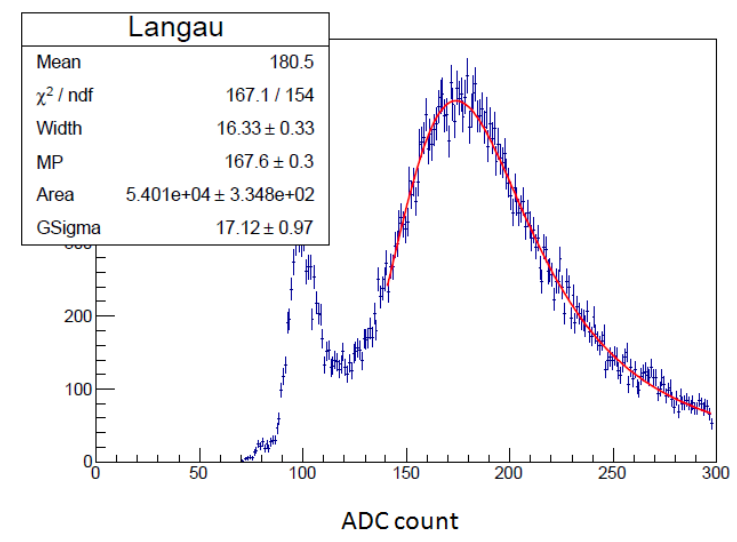

Figure 3: An example of the charge distribution of single muons obtained by PMTs. In this figure, charge of 1 ADC count corresponds to about $0.2 \mathrm{pC}$ and corresponds to about 4.5 photo electrons (p.e.) from the photocathode of PMT. Coincidence trigger of upper with lower layer of scintillators was applied for the data taking. Average value of the ADC counts in the pedestal run is $81.26 \pm 0.01$ in this figure. Convoluted Landau and Gaussian fitting function is used for the practical evaluation of the distribution. MP corresponds to the most probable parameter in Landau density. The difference between this parameter and the average value of the pedestal run is used for the evaluation of the signals. In this figure, MP corresponds to about 19.2 photo electrons. 


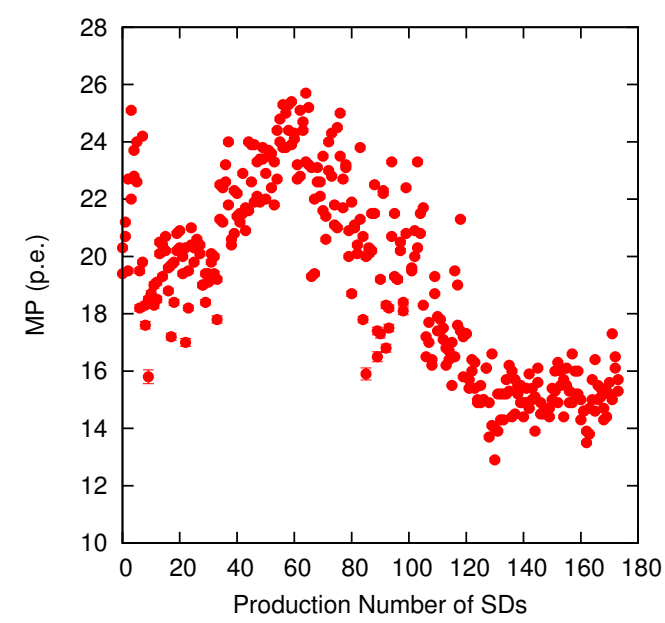

Figure 4: Distribution of MP (see the caption of Fig. 3) fitted to the obtained charge distribution of single muons with TAx4 SDs. The error bars show the fitting errors. The average of MP corresponds to 19 photo electrons and the root mean square is 3.3. The dependence of the production number of TAx 4 SDs comes from the light yield of the plastic scintillators.

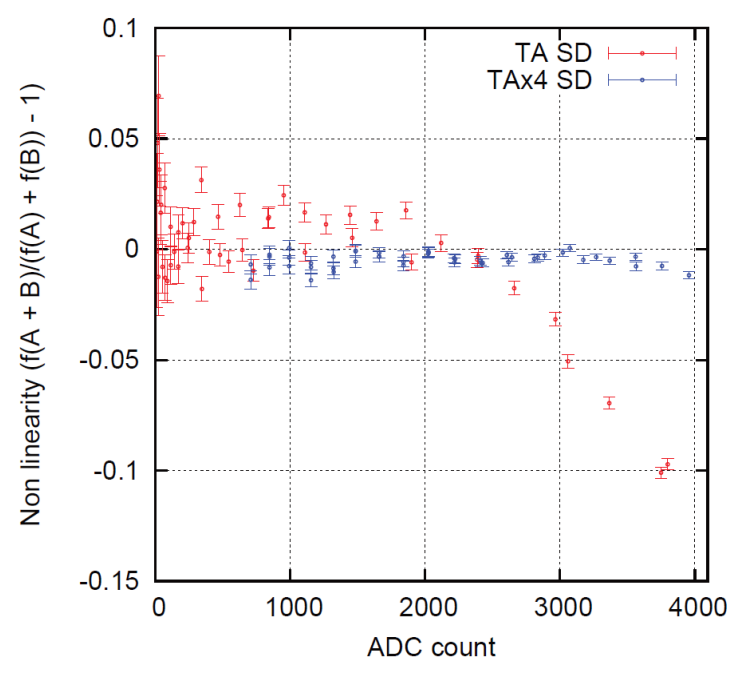

Figure 5: The measured linearity of typical PMTs for TA and TAx4 SDs. The statistical errors are shown as the error bars. Linearity was measured using LEDs which were instrumented in SDs. Y-axis means non-linearity of output current of PMTs. 1 ADC count corresponds to about $0.01 \mathrm{~mA}$. 

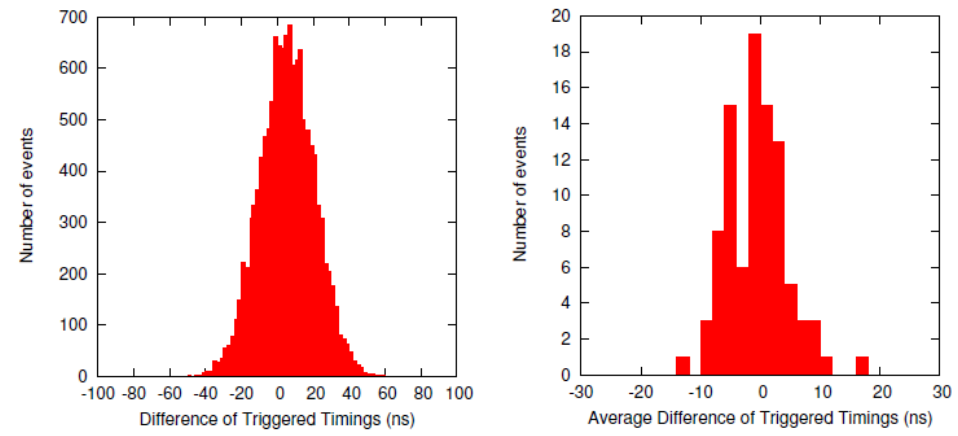

Figure 6: One test pulse was divided and the same divided pulses were input to some SD electronics and the difference of the trigger timings between SD electronics was measured. The source of the difference is the GPS timing receivers (M12M i-Lotus) which are instrumented on SD electronics. In the left panel, an example of the distribution of the relative trigger timings between 2 SD electronics is shown. The average of the trigger timings is $5.4 \mathrm{~ns}$ and the root mean square is $13.4 \mathrm{~ns}$. In the right panel, the average of trigger timings of 93 different GPS timing receivers compared with 1 standard GPS timing receiver are shown. The average of the histogram is $0.6 \mathrm{~ns}$ and the root mean square is $4.9 \mathrm{~ns}$. The largest data is $16.7 \mathrm{~ns}$.

done in 2017. TAx4 SDs will be deployed in the TAx4 SD site in winter 2017. The construction of TAx4 FDs will be started also in 2017. The assembled TAx4 SDs seem to realize the expected data quality for now. These detectors will enables us to study highest energies in more detail in the near future. 


\section{Acknowledgements}

The Telescope Array experiment is supported by the Japan Society for the Promotion of Science through Grants-in-Aids for Scientific Research on Specially Promoted Research (15H05693) and for Scientific Research (S) (15H05741), and the Inter-University Research Program of the Institute for Cosmic Ray Research; by the U.S. National Science Foundation awards PHY-0307098, PHY-0601915, PHY-0649681, PHY-0703893, PHY-0758342, PHY-0848320, PHY-1069280, PHY1069286, PHY-1404495 and PHY-1404502; by the National Research Foundation of Korea (2015R1A2A1A01006870，2015R1A2A1A15055344，2016R1A5A1013277，2007-0093860, 2016R1A2B4014967); by the Russian Academy of Sciences, RFBR grant 16-02-00962a (INR), IISN project No. 4.4502.13, and Belgian Science Policy under IUAP VII/37 (ULB). The foundations of Dr. Ezekiel R. and Edna Wattis Dumke, Willard L. Eccles, and George S. and Dolores Doré Eccles all helped with generous donations. The State of Utah supported the project through its Economic Development Board, and the University of Utah through the Office of the Vice President for Research. The experimental site became available through the cooperation of the Utah School and Institutional Trust Lands Administration (SITLA), U.S. Bureau of Land Management (BLM), and the U.S. Air Force. We appreciate the assistance of the State of Utah and Fillmore offices of the BLM in crafting the Plan of Development for the site. We also wish to thank the people and the officials of Millard County, Utah for their steadfast and warm support. We gratefully acknowledge the contributions from the technical staffs of our home institutions. An allocation of computer time from the Center for High Performance Computing at the University of Utah is gratefully acknowledged.

\section{References}

[1] R. Abbasi et al., ApJ. 790, L21 (2014).

[2] T. Abu-Zayyad et al., NIM-A, 689, 87A (2012). 